\title{
Design Principle of Telluride-Based Nanowire Heterostructures for Potential Thermoelectric Applications
}

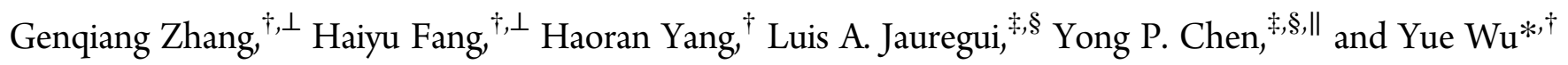 \\ ${ }^{\dagger}$ School of Chemical Engineering, ${ }^{\ddagger}$ School of Electrical and Computer Engineering, ${ }^{\S}$ Birck Nanotechnology Center, and ${ }^{\| D e p a r t m e n t}$ \\ of Physics, Purdue University, West Lafayette, Indiana 47907, United States
}

ABSTRACT: We present a design principle to develop new categories of telluride-based thermoelectric nanowire heterostructures through rational solution-phase reactions. The catalyst-free synthesis yields $\mathrm{Te}-\mathrm{Bi}_{2} \mathrm{Te}_{3}$ "barbell" nanowire heterostructures with a narrow diameter and length distribution as well as a rough control over the density of the hexagonal $\mathrm{Bi}_{2} \mathrm{Te}_{3}$ plates on the Te nanowire bodies, which can be further converted to other telluride-based compositionalmodulated nanowire heterostructures such as $\mathrm{PbTe}-\mathrm{Bi}_{2} \mathrm{Te}_{3}$. Initial characterizations of the hot-pressed nanostructured bulk pellets of the $\mathrm{Te}-\mathrm{Bi}_{2} \mathrm{Te}_{3}$ heterostructure show a largely enhanced Seebeck coefficient and greatly reduced thermal

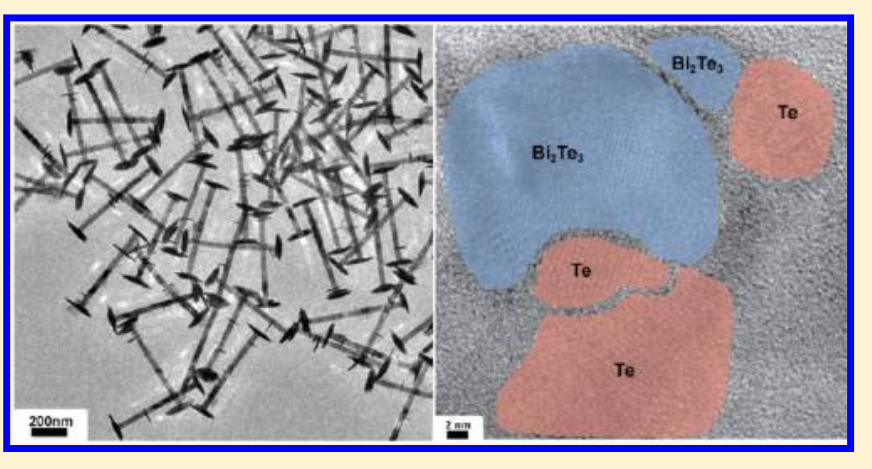
conductivity, which lead to an improved thermoelectric figure of merit. This approach opens up new platforms to investigate the phonon scattering and energy filtering.

KEYWORDS: Thermoelectric, tellurium, bismuth telluride, lead telluride, heterostructures

T hermoelectric (TE) devices, which can perform a direct conversion between thermal and electrical energy, have attracted great attention due to their promising potential in improving the energy efficiency and in solid-state cooling. ${ }^{1-5}$ However, the low efficiency of the TE materials prohibits their wide applications. Certain TE materials, such as $\mathrm{Bi}_{2} \mathrm{Te}_{3} / \mathrm{Sb}_{2} \mathrm{Te}_{3}$ superlattice film ${ }^{6}(\mathrm{ZT} \sim 2.4)$ and $\mathrm{AgPb}_{m} \mathrm{SbTe}_{2+m}$ bulk crystals ${ }^{7}$ (ZT 2.2), although possessing high performance due to the improved phonon scattering at nanoscale interfaces and grain boundaries, require very complicated material composition or an extremely expensive/time-consuming manufacture process such as molecular beam epitaxy. Theoretical predictions and initial experimental results have suggested that one-dimensional (1D) nanostructures, especially the nanowire heterostructures, which take the advantages of both quantum confinement to enhance the power factor and phonon scattering at nanowire surface and compositional interfaces to lower thermal conductivity, could offer a much higher ZT value. ${ }^{8-12}$ Meanwhile, the syntheses of various 1D nanowire heterostructures have been demonstrated through the chemical vapor deposition process based on vapor-liquid-solid (VLS) growth mechanism as well as the pulsed electrodeposition, ${ }^{13-18}$ but it is still a great challenge to obtain high-quality thermoelectric nanowire heterostructures in a simple yet scalable way.

We choose solution-phase chemical routes to explore the rational and scalable synthesis of $1 \mathrm{D}$ thermoelectric nanowire heterostructures with a particular focus on telluride-based compounds. Previously, there have been only few studies showing the growth of $\mathrm{Te}-\mathrm{Bi}_{2} \mathrm{Te}_{3}$ heterostructures with dimensions over hundreds of nanometers using expensive precursors and surfactant ${ }^{19}$ or through the catalyst-assisted growth of $\mathrm{Bi}_{2} \mathrm{Te}_{3}$ plates on the tips of Te nanorods, ${ }^{20}$ but the control of size/density of $\mathrm{Bi}_{2} \mathrm{Te}_{3}$ plates has not been demonstrated. In addition, the unintentional doping of catalyst cations $(\mathrm{Fe})$ could also alternate the intrinsic properties of thermoelectric materials. All of these have resulted in the unclearness of the thermoelectric performance and how such a heterogeneous system can be generally developed into other heterostructures. Herein, we show a catalyst-free synthesis of $\mathrm{Te}-\mathrm{Bi}_{2} \mathrm{Te}_{3}$ "barbell" nanowire heterostructures with a narrow diameter and length distribution as well as a rough control over the density of the hexagonal $\mathrm{Bi}_{2} \mathrm{Te}_{3}$ plates on the Te nanowire bodies by varying the reaction conditions. We also demonstrate the further conversion to other telluride-based compositionalmodulated nanowire heterostructures such as $\mathrm{PbTe}-\mathrm{Bi}_{2} \mathrm{Te}_{3}$. The initial characterizations of the hot-pressed nanostructured bulk pellets made from the $\mathrm{Te}-\mathrm{Bi}_{2} \mathrm{Te}_{3}$ heterostructures show a largely enhanced Seebeck coefficient and greatly reduced thermal conductivity, leading to an enhanced thermoelectric figure of merit.

The synthesis of $\mathrm{Te}-\mathrm{Bi}_{2} \mathrm{Te}_{3}$ "barbell" nanowire heterostructures is carried out in a standard Schlenk line with nitrogen protection. Tellurium dioxide $\left(\mathrm{TeO}_{2}, 99 \%+\right)$, ethylene glycol (EG, 99\%+), potassium hydroxide flakes (KOH, 90\%), polyvinylpyrrolidone (PVP, average molecule weight 40000 ), hydrazine hydrate solution $\left(\mathrm{N}_{2} \mathrm{H}_{4} \cdot \mathrm{H}_{2} \mathrm{O}, 80 \%\right)$, bismuth nitrate pentahydrate $\left(\mathrm{Bi}\left(\mathrm{NO}_{3}\right)_{3} \cdot 5 \mathrm{H}_{2} \mathrm{O}, 98 \%\right)$, and lead acetate

Received: April 8, 2012

Revised: June 15, 2012

Published: June 25, 2012 


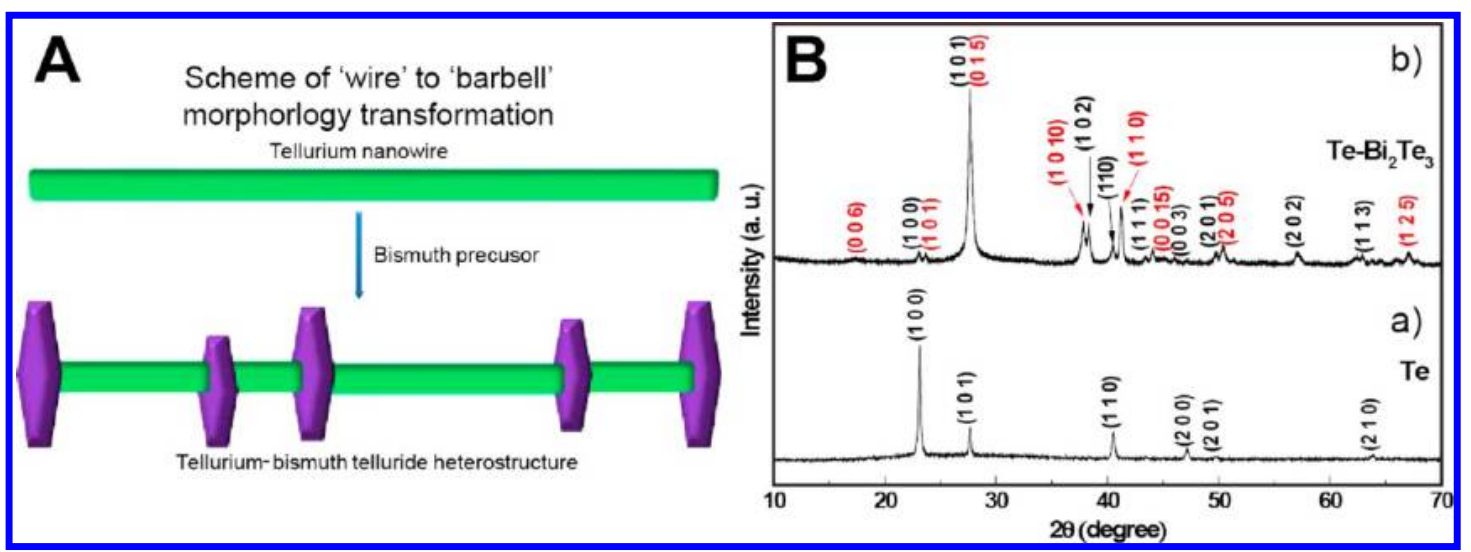

Figure 1. (A) Schematic of tellurium nanowire formed in the first step and tellurium-bismuth telluride heterostructure after adding bismuth precursor in the second step. (B) X-ray diffraction (XRD) patterns of Te nanowires and $\mathrm{Te}-\mathrm{Bi}_{2} \mathrm{Te}_{3}$ heterogeneous nanostructures after the injection of $\mathrm{Bi}$ precursor solution (the black marks stand for the peaks from $\mathrm{Te}$, and the red stand for $\mathrm{Bi}_{2} \mathrm{Te}_{3}$ ).

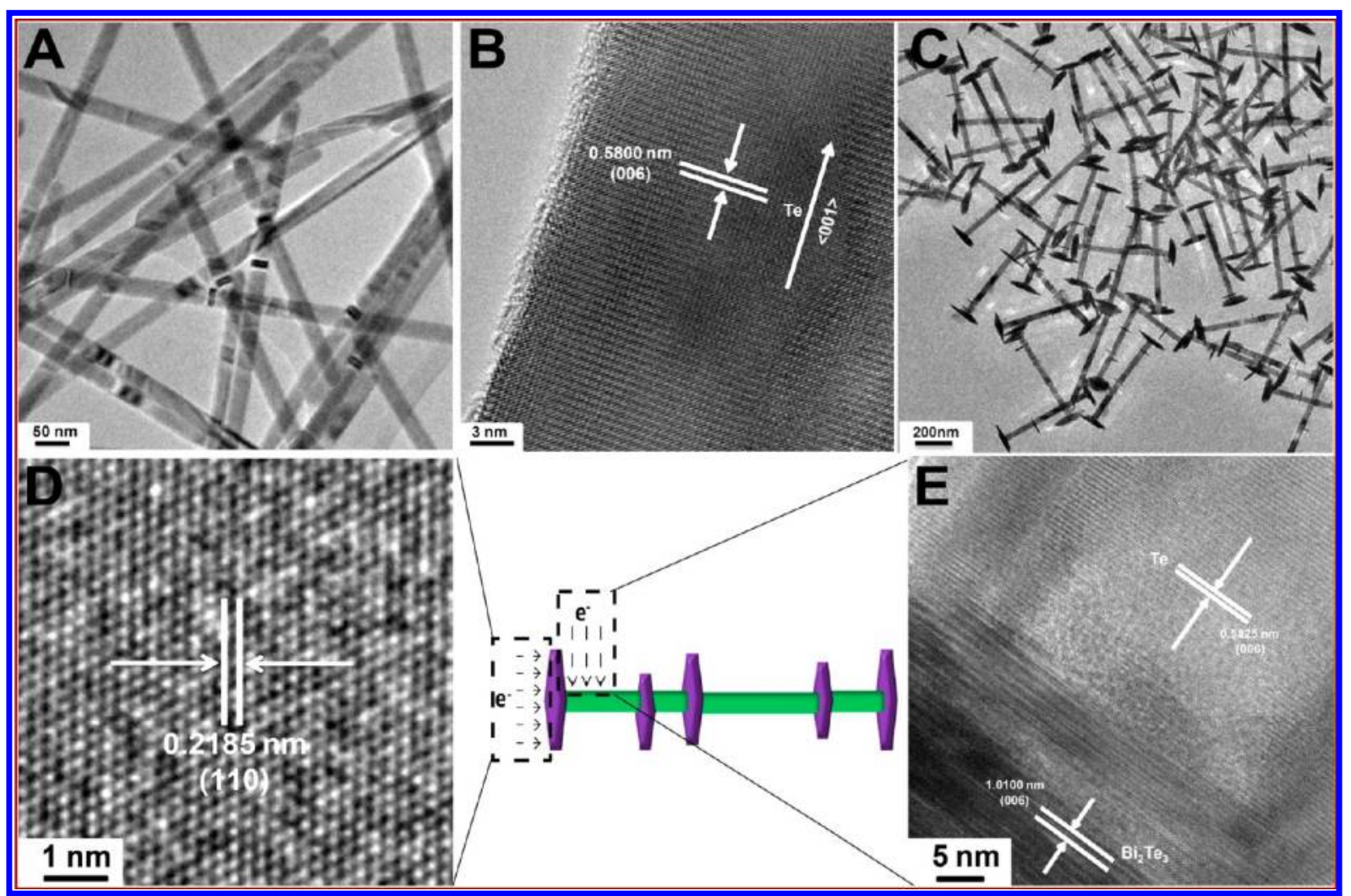

Figure 2. TEM images of Te nanowires and $\mathrm{Te}-\mathrm{Bi}_{2} \mathrm{Te}_{3}$ nanowire-multiple plates heterostructure: (A) low magnification and (B) HRTEM images of tellurium nanowire; (C) low magnification and (D, E) HRTEM images of the $\mathrm{Te}-\mathrm{Bi}_{2} \mathrm{Te}_{3}$ heterostructure. The scheme indicates the regions/view directions studied by HRTEM. Part $\mathrm{D}$ shows the top view of the $\mathrm{Bi}_{2} \mathrm{Te}_{3}$ plate, and part $\mathrm{E}$ shows the side view of $\mathrm{Bi}_{2} \mathrm{Te}_{3}$ plate and the junction between $\mathrm{Te}$ and $\mathrm{Bi}_{2} \mathrm{Te}_{3}$.

trihydrate $\left.\left(\mathrm{Pb}\left(\mathrm{CH}_{3} \mathrm{CO}_{2}\right)_{2} \cdot 3 \mathrm{H}_{2} \mathrm{O}, 99 \%+\right)\right)$ were all purchased from Sigma-Aldrich. All of the chemicals are used as received without further purification. In a typical synthesis, $1.5 \mathrm{mmol}$ of $\mathrm{TeO}_{2}, 10 \mathrm{mmol}$ of $\mathrm{KOH}, 0.3 \mathrm{~g}$ of PVP, and $15 \mathrm{~mL}$ of EG are added into a $50 \mathrm{~mL}$ three-neck flask. Nitrogen is purged through the system to keep the reaction in an oxygen-free environment. The mixture is stirred and heated to $140{ }^{\circ} \mathrm{C}$. After all of the chemicals are dissolved thoroughly, $0.15 \mathrm{~mL}$ of $80 \%$ hydrazine hydrate solution is injected into the reaction, and the yellow-transparent solution starts to turn into black slurry, which is kept under $140{ }^{\circ} \mathrm{C}$ for $1 \mathrm{~h}$ to let Te nanowires form completely. Meanwhile, $0.5 \mathrm{mmol}$ of $\mathrm{Bi}\left(\mathrm{NO}_{3}\right)_{3} \cdot 5 \mathrm{H}_{2} \mathrm{O}$ is added into $5 \mathrm{~mL}$ of EG in a glass vial to form a solution, which is kept at $100-120^{\circ} \mathrm{C}$. After one hour, the temperature of Te nanowire solution is raised to $160{ }^{\circ} \mathrm{C}$, and the $\mathrm{Bi}$ $\left(\mathrm{NO}_{3}\right)_{3} \cdot 5 \mathrm{H}_{2} \mathrm{O} / \mathrm{EG}$ solution is hot-injected into the flask. The reaction continues at $160{ }^{\circ} \mathrm{C}$ for $1 \mathrm{~h}$, and then the solution is cooled down naturally and the product is centrifuged followed by washing with deionized water three times and ethanol twice. The whole process is shown in Figure 1A.

The intermediate product of $\mathrm{Te}$ nanowires and the $\mathrm{Te}-$ $\mathrm{Bi}_{2} \mathrm{Te}_{3}$ "barbell" nanowire heterostructures are first characterized using X-ray diffraction (XRD). The lower spectrum in Figure $1 \mathrm{~B}$ can be readily indexed to pure hexagonal phase $\mathrm{Te}$ 


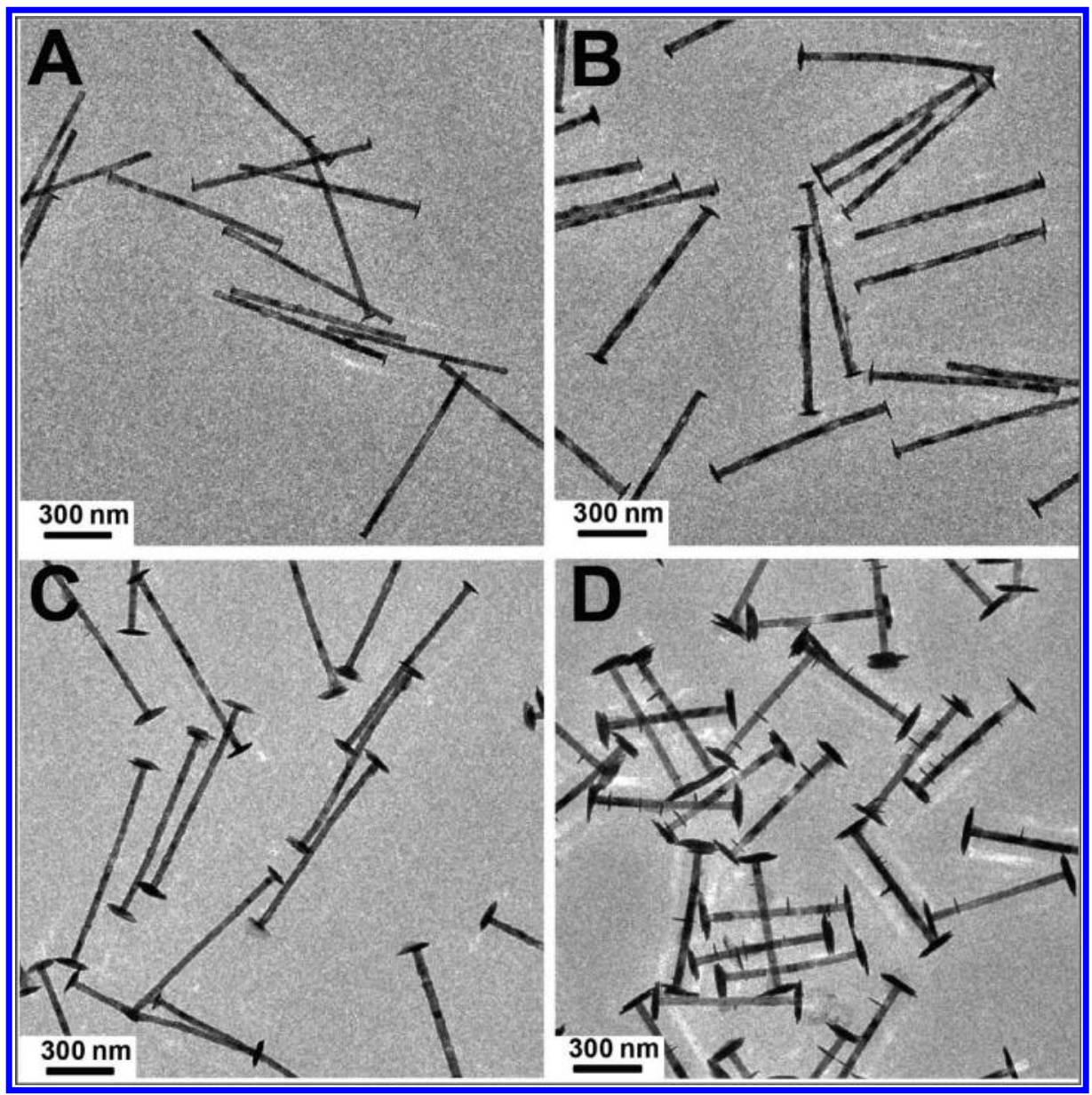

Figure 3. Evolution of "barbell" morphology by adding different amounts of hydrazine hydrate in the reaction: (A) $0.6 \mathrm{~mL}$; (B) $0.5 \mathrm{~mL}$; (C) $0.4 \mathrm{~mL}$; and (D) $0.3 \mathrm{~mL}$.

(JCPDS No. 36-1452), while the upper spectrum clearly indicates the partial formation of $\mathrm{Bi}_{2} \mathrm{Te}_{3}$ (JCPDS No. 15-0863) after adding the $\mathrm{Bi}$ precursor. Transmission electron microscopy (TEM) studies further confirm the conversion from the Te nanowires (Figure $2 \mathrm{~A}$ and $\mathrm{B}$ ) to the "barbell" nanowire heterostructures of $\mathrm{Te}-\mathrm{Bi}_{2} \mathrm{Te}_{3}$ (Figure 2C-E) and reveal several important features: First, the TEM data demonstrate clearly the uniformity of the $\mathrm{Te}$ nanowires and $\mathrm{Te}-\mathrm{Bi}_{2} \mathrm{Te}_{3}$ nanowire heterostructures. Statistic analyses performed on Figure $2 \mathrm{~A}$ and $\mathrm{C}$ show that the diameter of Te nanowires and the $\mathrm{Te}$ parts in the $\mathrm{Te}-\mathrm{Bi}_{2} \mathrm{Te}_{3}$ nanowire heterostructures are $36.62 \pm 1.46 \mathrm{~nm}$ and $36.92 \pm 1.86 \mathrm{~nm}$, respectively. The nearly unchanged diameters suggest that the growth of $\mathrm{Bi}_{2} \mathrm{Te}_{3}$ is highly selective. The random deposition and alloying over the $\mathrm{Te}$ nanowire body in our two-step reaction is strongly suppressed, which usually will result in an obvious change in diameter as observed in our previous report; ${ }^{21}$ Second, highresolution TEM (HRTEM, Figure 2B,D,E) studies show the lattice-resolved images and prove that both $\mathrm{Te}$ nanowires and $\mathrm{Te}-\mathrm{Bi}_{2} \mathrm{Te}_{3}$ nanowire heterostructures are single crystals. The distance between two neighboring lattice fringes in Figure $2 \mathrm{~B}$ is about $0.58 \mathrm{~nm}$, corresponding to the $\mathrm{Te}(006)$ crystal planes and suggesting the growth direction for Te nanowires is along $c$-axis, which is mainly due to the anisotropic crystal structure in hexagonal Te phase. ${ }^{22,23}$ Figure 2 parts $\mathrm{D}$ and $\mathrm{E}$ show the top view (Figure $2 \mathrm{D}$ ) of the $\mathrm{Bi}_{2} \mathrm{Te}_{3}$ plate and the side view (Figure 2E) of $\mathrm{Bi}_{2} \mathrm{Te}_{3}$ plate and the junction between $\mathrm{Te}$ and $\mathrm{Bi}_{2} \mathrm{Te}_{3}$ in the $\mathrm{Te}-\mathrm{Bi}_{2} \mathrm{Te}_{3}$ nanowire heterostructures along the view directions highlighted in the scheme. The lattice fringes with a distance of $0.2185 \mathrm{~nm}$ in Figure 2D correspond to the (110) crystal planes of $\mathrm{Bi}_{2} \mathrm{Te}_{3}$ phase. The side view (Figure $2 \mathrm{E}$ ) of $\mathrm{Bi}_{2} \mathrm{Te}_{3}$ plate shows the lattice fringe of $\mathrm{Bi}_{2} \mathrm{Te}_{3}(006)$ crystal planes and the epitaxial growth interface between $\mathrm{Te}$ and $\mathrm{Bi}_{2} \mathrm{Te}_{3}$, which is mainly due to the small lattice mismatch (as low as $1.62 \%)$ between the (001) crystal directions of $\mathrm{Te}$ and $\mathrm{Bi}_{2} \mathrm{Te}_{3}{ }^{19,24}$

Notably, the concentration and the amount of hydrazine have been found to significantly impact the composition of the final product as well as the size and the density of the Te nanowires and $\mathrm{Bi}_{2} \mathrm{Te}_{3}$ plates. Using anhydrous hydrazine in the reaction will only lead to the formation of pure $\mathrm{Bi}_{2} \mathrm{Te}_{3}$ nanowires with a much thinner diameter, which is consistent with our previous report. ${ }^{21}$ Decreasing the amount of $80 \%$ hydrazine hydrate added into the reaction (Figure 3 ) leads to the growth of a thicker Te nanowire body with larger $\mathrm{Bi}_{2} \mathrm{Te}_{3}$ plates and promotes multiple nucleation and growth of $\mathrm{Bi}_{2} \mathrm{Te}_{3}$ plates along the surface of $\mathrm{Te}$ nanowires (Figure 3D). Examining and understanding these observations suggest a possible growth mechanism for the "barbell" heterostructure: it has been widely accepted that the tips of nanowires usually possess the highest reactivity where the reaction/growth tends to happen first. ${ }^{25,26}$ If anhydrous hydrazine is used, the strong reduction environment as well as the high transient concentration of bismuth atoms (reduced from $\mathrm{Bi}$ $\left(\mathrm{NO}_{3}\right)_{3} \cdot 5 \mathrm{H}_{2} \mathrm{O}$ by hydrazine) will override the preferred growth on Te nanowire tips so that a nonselective absorption and 
alloying between $\mathrm{Bi}$ and $\mathrm{Te}$ nanowire will lead to the uniform conversion into $\mathrm{Bi}_{2} \mathrm{Te}_{3}$ nanowires, which has been identified in the previous research. ${ }^{20,21}$ Reducing the concentration of hydrazine to $80 \%$ and using smaller and smaller amounts slows down the generation of $\mathrm{Bi}$ atoms, thus promoting the selective growth of $\mathrm{Bi}_{2} \mathrm{Te}_{3}$ plates on the $\mathrm{Te}$ nanowire tips. However, when the amount/concentration of hydrazine is below a certain threshold $(0.3 \mathrm{~mL})$, the reaction is now controlled by the diffusion because of the slow generation of $\mathrm{Bi}$ atoms, and the randomly absorbed $\mathrm{Bi}$ atoms on $\mathrm{Te}$ nanowire bodies will form isolated $\mathrm{Bi}_{2} \mathrm{Te}_{3}$ islands, which act as the new nucleation sites to guide the growth to follow the VolmerWeber model. ${ }^{27}$ The formation of these three-dimensional islands, along with coarsening, will cause multiple $\mathrm{Bi}_{2} \mathrm{Te}_{3}$ plates to grow on the Te nanowire surface. Statistic result from Figure $4 \mathrm{E}$ gives an average of $4.186 \pm 1.314 \mathrm{Bi}_{2} \mathrm{Te}_{3}$ plates per

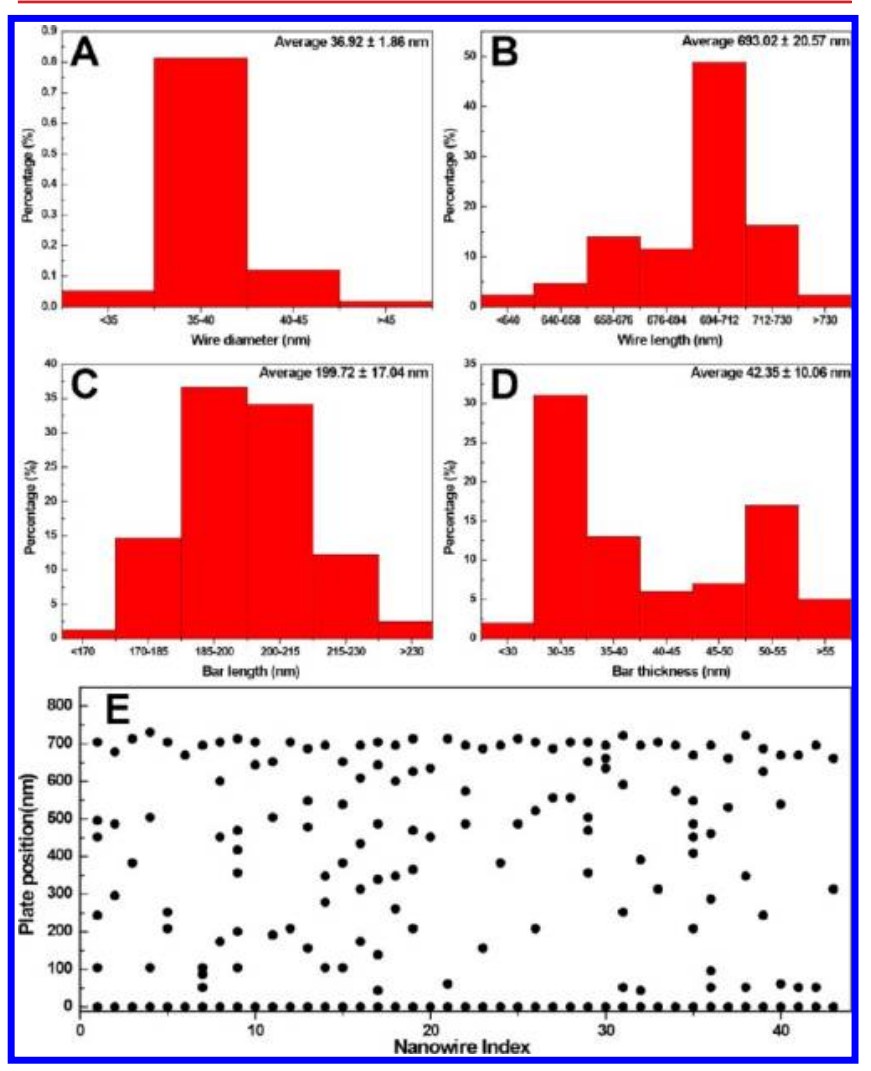

Figure 4. (A and B) Distribution of wire diameter and length; (C and D) distribution of bar length and thickness at the two ends of the wires; the reason for the two peaks in $\mathrm{D}$ is because two plates pile up at the ends of some wires; (E) the positions of the $\mathrm{Bi}_{2} \mathrm{Te}_{3}$ plates (black dots) on the nanowire heterostructures; the dots lined up perpendicularly to the $x$-axis are on a single wire.

nanowire. Notably, analysis of the structure parameters of the "barbell" heterostructures prepared using the conditions described in Figure 3D, especially the positions of the $\mathrm{Bi}_{2} \mathrm{Te}_{3}$ plates (the black dots in Figure 4E) in the nanowire heterostructures, indicates that the positions of isolated $\mathrm{Bi}_{2} \mathrm{Te}_{3}$ plates on the nanowire body is totally random, which is significantly different from other mechanisms such as lattice strain-induced heterostructure formation ${ }^{28}$ and further confirms the different growth mechanisms for the $\mathrm{Bi}_{2} \mathrm{Te}_{3}$ plates on $\mathrm{Te}$ nanowire tips and bodies.
Figure 4 shows the size distributions in the diameter (Figure $4 \mathrm{~A}, \mathrm{Te}$ nanowire body) and the length (Figure 4B, overall length) of the $\mathrm{Te}-\mathrm{Bi}_{2} \mathrm{Te}_{3}$ "barbell" nanowire heterostructures as well as in the length (Figure 4C) and the thickness (Figure $4 \mathrm{D})$ of the $\mathrm{Bi}_{2} \mathrm{Te}_{3}$ plates. The uniformity in all dimensions gives us a reliable and reproducible platform to study its fundamental electrical and thermal properties. In a typical process, we first remove the capping ligands on nanowire heterostructures by combining the $\mathrm{Te}-\mathrm{Bi}_{2} \mathrm{Te}_{3}$ "barbell" nanowire heterostructures dispersed in ethanol with diluted hydrazine solution $(10 \%$ volume ratio) and stirring vigorously until all of the nanowires are precipitated. The supernatant is decanted, and the precipitate is washed with ethanol three times to remove hydrazine residual. After the hydrazine treatment, the nanowires are collected by centrifugation, dried in vacuum, and consolidated into bulk pellets with $1.25 \mathrm{~cm}$ in diameter and $0.25 \mathrm{~cm}$ in thickness by hot press at $423 \mathrm{~K}$ for 30 min under an axial pressure of $150 \mathrm{MPa}$. For thermoelectric property measurements between 300 and $400 \mathrm{~K}$, the pellets are cut into regular rectangular shapes and mechanically polished before the measurement of electrical conductivity, Seebeck coefficient, and thermal conductivity. The electrical conductivity is measured through a standard four-probe method with a maximum temperature fluctuation of $\pm 2 \mathrm{~K}$. The Seebeck coefficient is measured by bridging the sample between a heater and heat sink and testing the voltage difference between the hot and the cold sides with a maximum temperature fluctuation of $\pm 0.2 \mathrm{~K}$ and a voltage resolution of $50 \mathrm{nV}$. The thermal conductivity $(\kappa)$ is measured through thermal diffusivity $(\alpha)$ and specific heat $\left(C_{p}\right)$ and then calculated via the equation $\kappa=$ $\alpha \rho C_{p}$ ( $\rho$ is the density).

Figure 5 shows the thermoelectric properties of the nanowire heterostructure composites after the hot press. Figure 5A shows the cross section HRTEM image of the nanowire heterostructure composite in which the nanoscale grain boundaries have been well-preserved to enhance the phonon scattering. The random orientations of the $\mathrm{Te}$ and $\mathrm{Bi}_{2} \mathrm{Te}_{3}$ domains also suggest the nanocomposite is a highly isotropic system. The electrical conductivity (Figure $5 \mathrm{~B}$ ) of the $\mathrm{Te}-\mathrm{Bi}_{2} \mathrm{Te}_{3}$ nanowire heterostructures increases from $3.051 \mathrm{~S} \cdot \mathrm{cm}^{-1}$ at $300 \mathrm{~K}$ to 5.244 $\mathrm{S} \cdot \mathrm{cm}^{-1}$ at $400 \mathrm{~K}$. Figure $5 \mathrm{C}$ shows the temperature dependence of Seebeck coefficient of the $\mathrm{Te}-\mathrm{Bi}_{2} \mathrm{Te}_{3}$ nanowire heterostructures. The positive Seebeck coefficient value indicates the p-type conduction. The Seebeck coefficient measurement shows a decreasing trend from $608 \mu \mathrm{V} \cdot \mathrm{K}^{-1}$ at $300 \mathrm{~K}$ to 588 $\mu \mathrm{V} \cdot \mathrm{K}^{-1}$ at $400 \mathrm{~K}$. The thermal conductivity (Figure $5 \mathrm{E}$ ) is measured to be $0.365 \mathrm{~W} \cdot \mathrm{m}^{-1} \cdot \mathrm{K}^{-1}$ at $300 \mathrm{~K}$ and decreases to $0.309 \mathrm{~W} \cdot \mathrm{m}^{-1} \cdot \mathrm{K}^{-1}$ at $400 \mathrm{~K}$. The calculated $\mathrm{ZT}$ for the Te$\mathrm{Bi}_{2} \mathrm{Te}_{3}$ nanowire heterostructures (Figure 5F) increases from 0.09 at $300 \mathrm{~K}$ to around 0.24 at $400 \mathrm{~K}$.

An analysis of these results highlights some important points: First, the electrical conductivity of our $\mathrm{Te}-\mathrm{Bi}_{2} \mathrm{Te}_{3}$ nanowire heterostructures $(3.051 \mathrm{~S} / \mathrm{cm}$ at $300 \mathrm{~K})$ is much higher than the value of the Te nanowires $(0.08 \mathrm{~S} / \mathrm{m} \text { at } 298 \mathrm{~K})^{29}$ and is comparable with the value of bulk Te $(3.04 \mathrm{~S} / \mathrm{m}$ at $293 \mathrm{~K})$. This improvement is mainly due to the hot press to form a nanostructured bulk disk with a reasonably high relative density $(\sim 63 \%)$ as well as the introduction of more electrically conductive $\mathrm{Bi}_{2} \mathrm{Te}_{3}$ to form the heterostructures, which through our rough estimation, counts for about $63.6 \%$ in the total volume. Further improvement in electrical conductivity could be achieved by optimizing the hot press temperature and pressure, and related systematic studies are underway. Second, 


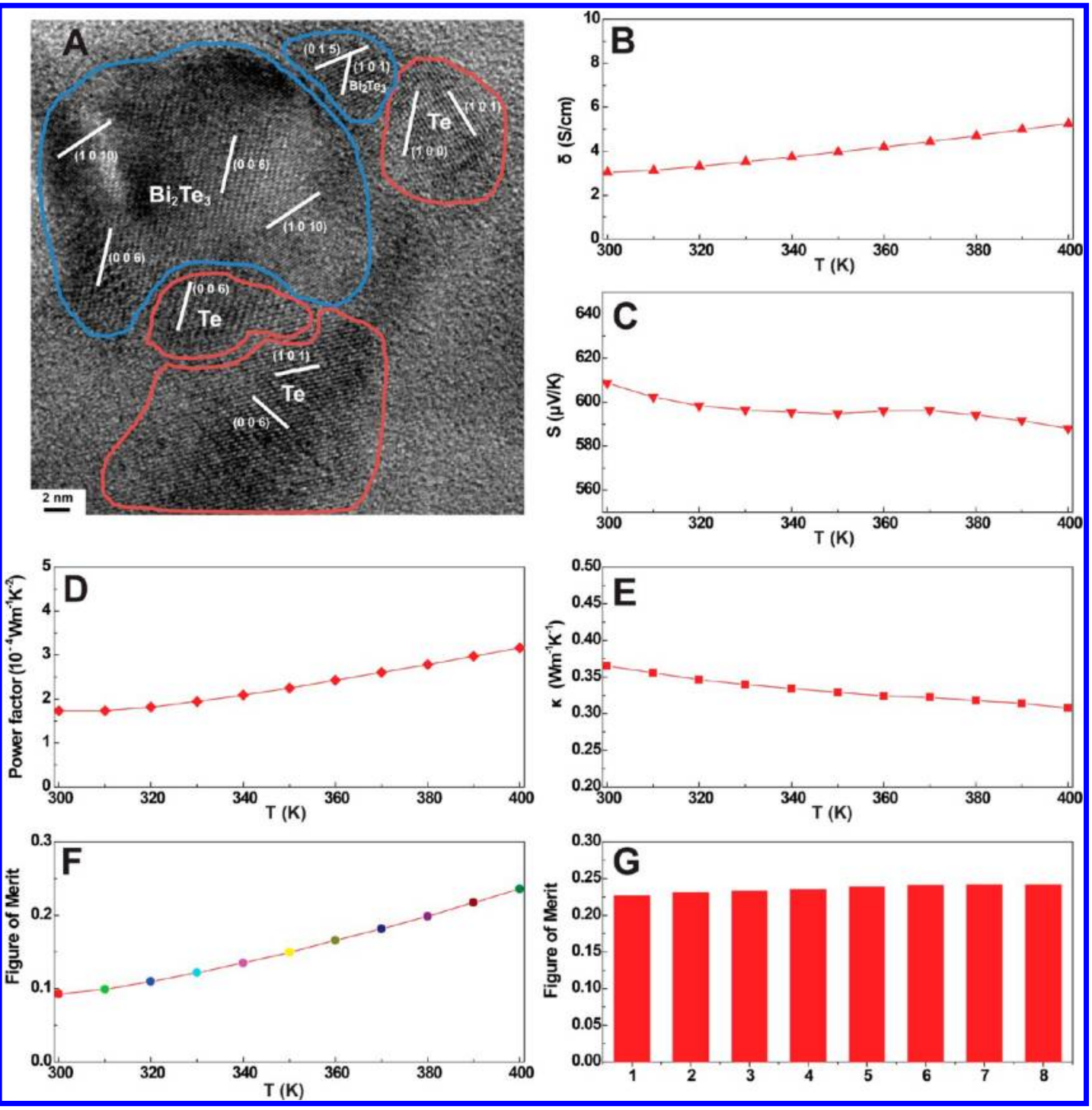

Figure 5. Thermoelectric properties of bulk nanocomposite pellet made by hot pressing the as-obtained $\mathrm{Te}-\mathrm{Bi}_{2} \mathrm{Te}_{3}$ nanowire heterostructures. (A) Cross section HRTEM image of hot-pressed sample which clearly shows nanoscale grain boundaries preserved inside the sample; (B) electric conductivity, (C) Seebeck coefficient, (D) power factor, (E) thermal conductivity, (F) ZT of a typical sample measured between 300 and $400 \mathrm{~K}$, and (G) the distribution of peak ZT from different samples.

the Seebeck coefficient in our $\mathrm{Te}-\mathrm{Bi}_{2} \mathrm{Te}_{3}$ nanowire heterostructures $\left(608 \mu \mathrm{V} \cdot \mathrm{K}^{-1}\right.$ at $300 \mathrm{~K}$ and $588 \mu \mathrm{V} \cdot \mathrm{K}^{-1}$ at $\left.400 \mathrm{~K}\right)$ is also considerably higher than that of $\mathrm{Te}$ nanowires (408 $\mu \mathrm{V} \cdot \mathrm{K}^{-1}$ at $\left.298 \mathrm{~K}\right),{ }^{29}$ Te bulk crystals $\left(340 \mu \mathrm{V} \cdot \mathrm{K}^{-1}\right.$ at $\left.293 \mathrm{~K}\right)$, and pure $\mathrm{Bi}_{2} \mathrm{Te}_{3}$ nanowires reported previously by our group $\left(205 \mu \mathrm{V} \cdot \mathrm{K}^{-1}\right.$ at $300 \mathrm{~K}$ and $245 \mu \mathrm{V} \cdot \mathrm{K}^{-1}$ at $\left.400 \mathrm{~K}\right){ }^{21}$ The largely enhanced Seebeck coefficient could result from the energy filtering effect occurring at grain-grain interfaces, as seen in Figure 5A in our hot pressed samples. To decide whether there is any energy filtering effect happening in the heterostructure, the work function and band gap of tellurium and bismuth telluride need to be experimentally determined. However, if we use the work function and band gap reported in previous literatures $\left(4.95 \mathrm{eV}\right.$ and $0.3 \mathrm{eV}$ for tellurium ${ }^{30}$ and $5.30 \mathrm{eV}$ and $0.15 \mathrm{eV}$ for bismuth telluride, ${ }^{31}$ respectively), the Fermi level offset between the two materials is $0.35 \mathrm{eV}$, which is similar to the ones in previous papers where energy filtering was observed. ${ }^{30,32}$ Due to the small band gap of bismuth telluride $(0.15 \mathrm{eV})$, it is partially degenerate near the Fermi level. ${ }^{33}$ Based on the facts above, it is possible that low-energy carriers (holes in this case) are scattered by the energy barrier and highenergy carriers pass through with the proper band alignment in our heterostructure, thus leading to an increased power factor by theoretical prediction. ${ }^{34,35}$ Third, the thermal conductivity of our sample $\left(0.365 \mathrm{~W} \cdot \mathrm{m}^{-1} \cdot \mathrm{K}^{-1}\right.$ at $300 \mathrm{~K}$ and $0.309 \mathrm{~W} \cdot \mathrm{m}^{-1} \cdot \mathrm{K}^{-1}$ at $400 \mathrm{~K})$ is only $\sim 16 \%$ of bulk Te crystal $\left(2.27 \mathrm{~W} \cdot \mathrm{m}^{-1} \cdot \mathrm{K}^{-1}\right.$ at $293 \mathrm{~K})$ and $\sim 26 \%$ of pure $\mathrm{Bi}_{2} \mathrm{Te}_{3}$ nanowires reported previously $\left(1.42 \mathrm{~W} \cdot \mathrm{m}^{-1} \cdot \mathrm{K}^{-1}\right.$ at $300 \mathrm{~K}$ and $1.19 \mathrm{~W} \cdot \mathrm{m}^{-1} \cdot \mathrm{K}^{-1}$ at $400 \mathrm{~K}){ }^{21}$ Such a low thermal conductivity is comparable to the Te nanowire-poly(3,4-ethylenedioxythiophene):poly(styrenesulfonate) (PEDOT:PSS) composite (0.22-0.30 $\mathrm{W} \cdot \mathrm{m}^{-1} \cdot \mathrm{K}^{-1}$ at $\left.298 \mathrm{~K}\right)$ and pure organic PEDOT:PSS polymer $\left(0.24-0.29 \mathrm{~W} \cdot \mathrm{m}^{-1} \cdot \mathrm{K}^{-1}\right.$ at $\left.298 \mathrm{~K}\right),{ }^{29}$ which directly benefits from the enhanced phonon scattering at nanowire-nanowire, nanowire-plate, and plate-plate interfaces. Lastly and most importantly, the $\mathrm{ZT}$ of our $\mathrm{Te}-\mathrm{Bi}_{2} \mathrm{Te}_{3}$ nanowire heterostructure-based composite is more than two orders better than pure Te nanowires $(0.0004$ at $298 \mathrm{~K})$ and 2.4 times better than the Te nanowire-PEDOT:PSS composite (0.1 at $298 \mathrm{~K})$. Moreover, our ZT has a very narrow distribution (0.2360 \pm 


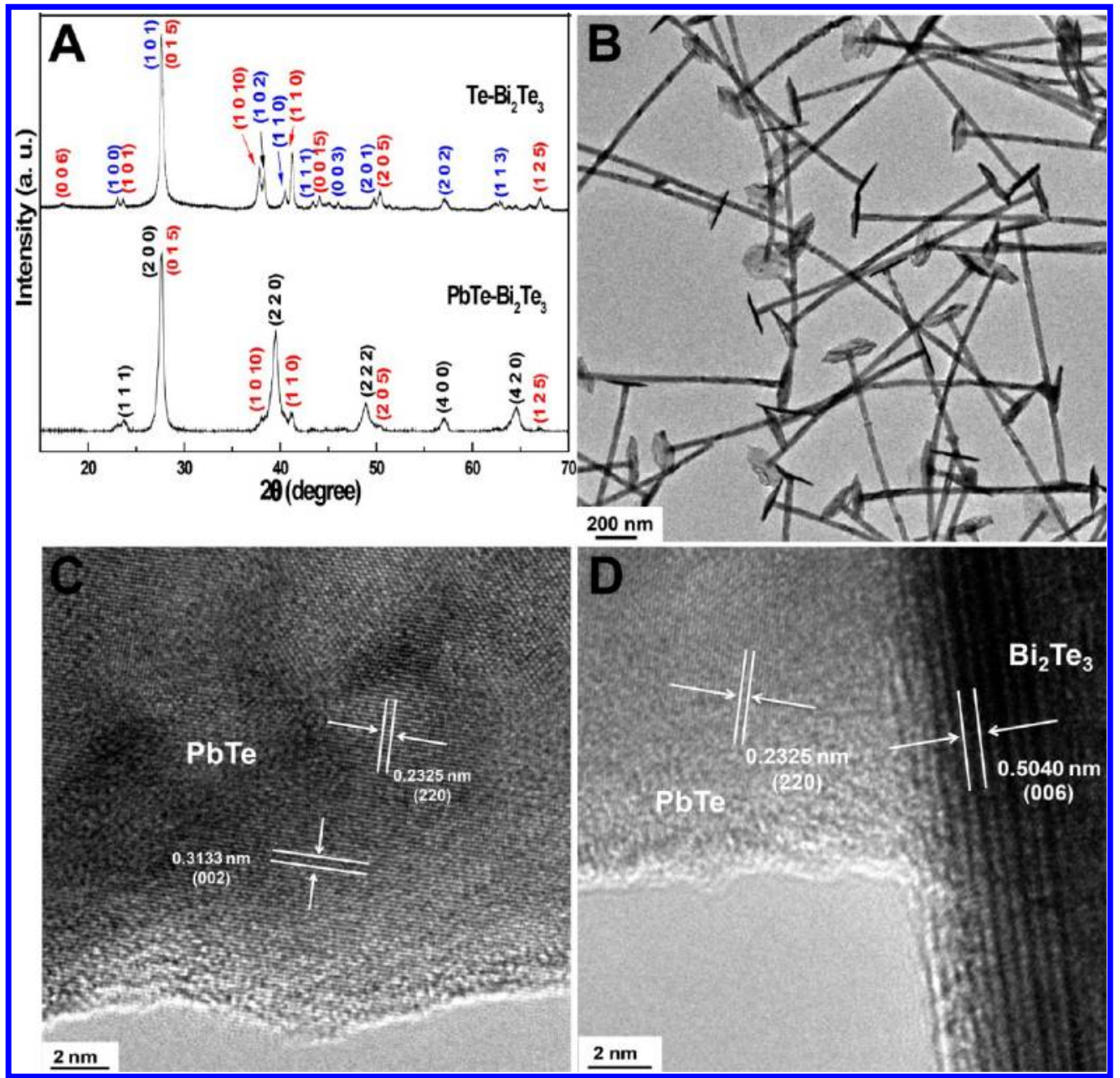

Figure 6. (A) XRD patterns of $\mathrm{Te}-\mathrm{Bi}_{2} \mathrm{Te}_{3}$ heterostructures and $\mathrm{PbTe}-\mathrm{Bi}_{2} \mathrm{Te}_{3}$ heterostructures after the injection of $\mathrm{Pb}$ precursor solution (the black marks stand for the peaks from $\mathrm{PbTe}$, the red for the ones from $\mathrm{Bi}_{2} \mathrm{Te}_{3}$, and the blue for the ones from $\mathrm{Te}$ ). (B) The TEM image of $\mathrm{PbTe}-$ $\mathrm{Bi}_{2} \mathrm{Te}_{3}$ barbell heterostructures. Parts $\mathrm{C}$ and $\mathrm{D}$ are the HRTEM images of PbTe nanowire body $(\mathrm{C})$ and the junction between PbTe nanowire body and $\mathrm{Bi}_{2} \mathrm{Te}_{3}$ plates $(\mathrm{D})$.

0.0057) as shown in Figure 5G, which further proves the reliability and reproducibility of our synthetic approach. Notably, the ZT value observed here is much lower compared to the pure $\mathrm{Bi}_{2} \mathrm{Te}_{3}$ nanowires $(0.96$ at $380 \mathrm{~K}){ }^{21}$ which is mainly due to the presence of large percentage of $\mathrm{Te}(\sim 36.4 \%)$ in the heterostructures, which significantly lowers the electrical conductivity of the heterostructures. ${ }^{21}$

The rational synthesis of $\mathrm{Te}-\mathrm{Bi}_{2} \mathrm{Te}_{3}$ nanowire heterostructures with high morphology, composition, and size uniformity provides a unique platform to design a whole family of telluride-based nanowire heterostructures. As an initial proof of concept, we explore the synthesis of $\mathrm{PbTe}-\mathrm{Bi}_{2} \mathrm{Te}_{3}$ nanowire heterostructures by selectively converting the $\mathrm{Te}$ regions in the $\mathrm{Te}-\mathrm{Bi}_{2} \mathrm{Te}_{3}$ nanowire heterostructures into $\mathrm{PbTe}$. This process involves the addition of an extra $0.4 \mathrm{~mL}$ of $80 \%$ hydrazine hydrate solution into the flask after the completion of the growth of $\mathrm{Bi}_{2} \mathrm{Te}_{3}$ plates at $160{ }^{\circ} \mathrm{C}$. The solution is stirred for $5 \mathrm{~min}$; then the stock solution of $\mathrm{Pb}$ precursor $(0.75 \mathrm{mmol}$ of $\mathrm{Pb}\left(\mathrm{CH}_{3} \mathrm{CO}_{2}\right)_{2} \cdot 3 \mathrm{H}_{2} \mathrm{O}$ dissolved in $5 \mathrm{~mL}$ of $\left.\mathrm{EG}\right)$ is injected into the solution, and the reaction continues at $160{ }^{\circ} \mathrm{C}$ for another hour. Figure 6 shows the structural characterizations of the $\mathrm{PbTe}-\mathrm{Bi}_{2} \mathrm{Te}_{3}$ nanowire heterostructures using X-ray diffraction (Figure 6A) and transmission electron microscopy (Figure 6B-D). The "barbell" heterostructure morphology is successfully retained during the transformation of $\mathrm{Te}$ into $\mathrm{PbTe}$ (Figure 6B) with both the $\mathrm{PbTe}$ regions (Figure 6C) and the $\mathrm{Bi}_{2} \mathrm{Te}_{3}$ plates (Figure $6 \mathrm{D}$ ) to be single crystal. The thermoelectric property of the $\mathrm{PbTe}-\mathrm{Bi}_{2} \mathrm{Te}_{3}$ nanowire heterostructures is still under investigation, and we believe this approach can be further generalized to prepare other telluride-based nanowire heterostructures, such as $\mathrm{Ag}_{2} \mathrm{Te}-\mathrm{Bi}_{2} \mathrm{Te}_{3}, \mathrm{Sb}_{2} \mathrm{Te}_{3}-$ $\mathrm{Bi}_{2} \mathrm{Te}_{3}, \mathrm{Cu}_{2} \mathrm{Te}-\mathrm{Bi}_{2} \mathrm{Te}_{3}, \mathrm{SnTe}-\mathrm{Bi}_{2} \mathrm{Te}_{3}$, and so forth.

In summary, we have successfully developed a rational solution phase synthetic approach that will instantly open up great wealth of opportunities for the fundamental studies about the electron and phonon interactions in the unique platforms of telluride-based nanowire heterostructures. Initial physical characterizations demonstrate a significantly improved thermoelectric performance due to the enhanced phonon scattering at nanowire heterostructure surface and interface, which could significantly inspire further advances in using novel nanowire heterostructures for thermoelectric energy conversion.

\section{AUTHOR INFORMATION}

\section{Corresponding Author}

*Tel.: +1-765-494-6028. E-mail: yuewu@purdue.edu. Fax: +1765-494-0805. 


\section{Author Contributions}

${ }^{\perp}$ These authors contributed equally to this work.

\section{Notes}

The authors declare no competing financial interest.

\section{ACKNOWLEDGMENTS}

Y.W. thanks the support from DuPont Young Faculty Award, AFOSR (Award Number FA9550-12-1-0061), and the Kaiteki Institute, Inc. Y.P.C. thanks the Intel Corporation. Y.W. acknowledges the use of the hot press setup at Prof. ChinTeh Sun's group at Purdue University.

\section{REFERENCES}

(1) Snyder, G. J.; Toberer, E. S. Nat. Mater. 2008, 7, 105.

(2) Dresselhaus, M. S.; Chen, G.; Tang, M. Y.; Yang, R. G.; Lee, H.; Wang, D. Z.; Ren, Z. F.; Fleurial, J. P.; Gogna, P. Adv. Mater. 2007, 19, 1043.

(3) Sales, B. C. Science 2002, 295, 1248.

(4) Bell, L. E. Science 2008, 321, 1457.

(5) Zhang, G. Q.; Yu, Q. X.; Wang, W.; Li, X. G. Adv. Mater. 2010, 22, 1959.

(6) Venkatasubramanian, R.; Siivola, E.; Colpitts, T.; O'Quinn, B. Nature 2001, 413, 597.

(7) Hsu, K. F.; Loo, S.; Guo, F.; Chen, W.; Dyck, J. S.; Uher, C.; Hogan, T.; Polychroniadis, E. K.; Kanatzidis, M. G. Science 2004, 303, 818.

(8) Hicks, L. D.; Dresselhaus, M. S. Phys. Rev. B 1993, 47, 16631.

(9) Lin, Y. M.; Dresselhaus, M. S. Phys. Rev. B 2003, 68.

(10) Dames, C.; Chen, G. J. Appl. Phys. 2004, 95, 682.

(11) Hochbaum, A. I.; Chen, R. K.; Delgado, R. D.; Liang, W. J.; Garnett, E. C.; Najarian, M.; Majumdar, A.; Yang, P. D. Nature 2008, $451,163$.

(12) Zhou, J. H.; Jin, C. G.; Seol, J. H.; Li, X. G.; Shi, L. Appl. Phys. Lett. 2005, 87, 133109.

(13) Wu, Y.; Xiang, J.; Yang, C.; Lu, W.; Lieber, C. M. Nature 2004, 430, 61 .

(14) Gudiksen, M. S.; Lauhon, L. J.; Wang, J.; Smith, D. C.; Lieber, C. M. Nature 2002, 415, 617.

(15) Wang, W.; Lu, X. L.; Zhang, T.; Zhang, G. Q.; Jiang, W. J.; Li, X. G. J. Am. Chem. Soc. 2007, 129, 6702.

(16) Wang, W.; Zhang, G. Q.; Li, X. G. J. Phys. Chem. C 2008, 112, 15190.

(17) Ouyang, L.; Maher, K. N.; Yu, C. L.; McCarty, J.; Park, H. J. Am. Chem. Soc. 2007, 129, 133.

(18) Jin, C. G.; Zhang, G. Q.; Qian, T.; Li, X. G.; Yao, Z. J. Phys. Chem. B 2005, 109, 1430.

(19) Lu, W.; Ding, Y.; Chen, Y.; Wang, Z. L.; Fang, J. J. Am. Chem. Soc. 2005, 127, 10112.

(20) Wang, W.; Goebl, J.; He, L.; Aloni, S.; Hu, Y.; Zhen, L.; Yin, Y. J. Am. Chem. Soc. 2010, 132, 17316.

(21) Zhang, G.; Kirk, B.; Jauregui, L. A.; Yang, H.; Xu, X.; Chen, Y. P.; Wu, Y. Nano Lett. 2012, 12, 56.

(22) Tang, Z. Y.; Wang, Y.; Sun, K.; Kotov, N. A. Adv. Mater. 2005, 17,358 .

(23) Zhang, B.; Hou, W. Y.; Ye, X. C.; Fu, S. Q.; Xie, Y. Adv. Funct. Mater. 2007, 17, 486.

(24) Habas, S. E.; Lee, H.; Radmilovic, V.; Somorjai, G. A.; Yang, P. Nat. Mater. 2007, 6, 692.

(25) Sadtler, B.; Demchenko, D. O.; Zheng, H.; Hughes, S. M.; Merkle, M. G.; Dahmen, U.; Wang, L.-W.; Alivisatos, A. P. J. Am. Chem. Soc. 2009, 131, 5285.

(26) Saunders, A. E.; Popov, I.; Banin, U. J. Phys. Chem. B 2006, 110, 5421

(27) Tian, X.; Du, S.; Gao, H. Chin. Phys. B 2008, 17, 286.

(28) Robinson, R. D.; Sadtler, B.; Demchenko, D. O.; Erdonmez, C. K.; Wang, L. W.; Alivisatos, A. P. Science 2007, 317, 355.
(29) See, K. C.; Feser, J. P.; Chen, C. E.; Majumdar, A.; Urban, J. J.; Segalman, R. A. Nano Lett. 2010, 10, 4664.

(30) Zhang, Y.; Snedaker, M. L. Nano Lett. 2012, 2, 1075.

(31) Haneman, D. J. Phys. Chem. Solids 1959, 11, 205.

(32) Ko, D. K.; Kang, Y.; Murray, C. B. Nano Lett. 2011, 11, 2841.

(33) Fleurial, J. P.; Gailliard, L.; Triboulet, R.; Scherrer, H.; Scherrer, S. J. Phys. Chem. Solids 1988, 49, 1237.

(34) Shakouri, A. Annu. Rev. Mater. Res. 2011, 41, 399.

(35) Vashaee, D.; Shakouri, A. Phys. Rev. Lett. 2004, 92, 106103. 